\title{
Establishment and application of a CRISPR- Cas 12 a assisted genome-editing system in Zymomonas mobilis
}

Wei Shen, Jun Zhang, Binan Geng, Mengyue Qiu, Mimi Hu, Qing Yang, Weiwei Bao, Yubei Xiao, Yanli Zheng, Wenfang Peng, Guimin Zhang, Lixin Ma and Shihui Yang* (D)

\begin{abstract}
Background: Efficient and convenient genome-editing toolkits can expedite genomic research and strain improvement for desirable phenotypes. Zymomonas mobilis is a highly efficient ethanol-producing bacterium with a small genome size and desirable industrial characteristics, which makes it a promising chassis for biorefinery and synthetic biology studies. While classical techniques for genetic manipulation are available for $Z$. mobilis, efficient genetic engineering toolkits enabling rapidly systematic and high-throughput genome editing in $Z$. mobilis are still lacking.
\end{abstract}

Results: Using Cas12a (Cpf1) from Francisella novicida, a recombinant strain with inducible cas 12a expression for genome editing was constructed in Z. mobilis ZM4, which can be used to mediate RNA-guided DNA cleavage at targeted genomic loci. gRNAs were then designed targeting the replicons of native plasmids of ZM4 with about 100\% curing efficiency for three native plasmids. In addition, CRISPR-Cas12a recombineering was used to promote gene deletion and insertion in one step efficiently and precisely with efficiency up to $90 \%$. Combined with single-stranded DNA (sSDNA), CRISPR-Cas12a system was also applied to introduce minor nucleotide modification precisely into the genome with high fidelity. Furthermore, the CRISPR-Cas12a system was employed to introduce a heterologous lactate dehydrogenase into $Z$. mobilis with a recombinant lactate-producing strain constructed.

Conclusions: This study applied CRISPR-Cas12a in Z. mobilis and established a genome editing tool for efficient and convenient genome engineering in Z. mobilis including plasmid curing, gene deletion and insertion, as well as nucleotide substitution, which can also be employed for metabolic engineering to help divert the carbon flux from ethanol production to other products such as lactate demonstrated in this work. The CRISPR-Cas12a system established in this study thus provides a versatile and powerful genome-editing tool in Z. mobilis for functional genomic research, strain improvement, as well as synthetic microbial chassis development for economic biochemical production.

Keywords: Zymomonas mobilis, CRISPR, Cas12a, ssDNA recombineering, In situ mutagenesis, Lactate, Genome engineering

\footnotetext{
${ }^{*}$ Correspondence: Shihui.Yang@hubu.edu.cn

State Key Laboratory of Biocatalysis and Enzyme Engineering,

Environmental Microbial Technology Center of Hubei Province

and School of Life Sciences, Hubei University, Wuhan 430062, China
}

(c) The Author(s) 2019. This article is distributed under the terms of the Creative Commons Attribution 4.0 International License (http://creativecommons.org/licenses/by/4.0/), which permits unrestricted use, distribution, and reproduction in any medium, provided you give appropriate credit to the original author(s) and the source, provide a link to the Creative Commons license, and indicate if changes were made. The Creative Commons Public Domain Dedication waiver (http://creativecommons.org/ publicdomain/zero/1.0/) applies to the data made available in this article, unless otherwise stated. 


\section{Introduction}

Zymomonas mobilis is a facultative anaerobic Gramnegative ethanologenic bacterium and generally regarded as safe (GRAS), which anaerobically ferments glucose, fructose and sucrose for ethanol production through Entner-Doudoroff (ED) pathway with many desirable characteristics, such as high specific glucose uptake rate, rapid catabolism, high tolerance of ethanol concentration up to $16 \%(\mathrm{v} / \mathrm{v})$ and broad $\mathrm{pH}$ range (4-7.5) [1-4]. The metabolically engineered recombinant $Z$. mobilis broadens the fermentable substrates to pentose sugars such as xylose and arabinose, which is a promising microorganism for economic biochemical production from lignocellulosic biomass [5-7]. Moreover, many systems biology studies of $Z$. mobilis have been carried out with significant omics datasets accumulated, providing valuable information for strain improvement [8-14].

The capability of systematic and high-throughput modifying microbial genome for desirable phenotypes represents a great advancement for fully understanding of gene function and regulatory networks [15]. Conventional genetic studies in Z. mobilis are usually based on homologous recombination (HR) using suicide or unstable replicative vectors. Currently, metabolic engineering and synthetic biology methods have been improved rapidly and various genetic engineering tools including suicide plasmid-based mutant construction, site-specific flippase (FLP) recombination and transposon mutagenesis, as well as RecET recombination system have been applied in Z. mobilis for gene function analysis and metabolic engineering $[7,16,17]$. Among these methods, the RecET recombination system derived from bacteriophage $\lambda$-Red system provides an efficient tool to induce homologous recombination between linear DNA fragments and bacterial chromosomes with the use of selectable markers. However, inevitable obstacles exist with respect to the capability of these traditional methods. For example, the allelic exchange methods involve either introducing a selectable marker into the edited locus or a counterselection system such as $\mathrm{SacB}$, which generally are low efficiency, time-consuming, and laborious [18]. Moreover, these systems are usually restricted by the available selection markers and the expression of the recombinase in the host $[19,20]$. Therefore, it is crucial to develop an efficient and convenient genetic engineering tool to expedite the strain development in Z. mobilis.

The Clustered Regularly Interspaced Short Palindromic Repeats (CRISPR)-CRISPR-associated (CRISPR-Cas) system is a widely distributed prokaryotic adaptive immunity system that confers resistance to invading genetic elements in archaea and bacteria [21-23]. CRISPR-Cas systems consist of CRISPR arrays and Cas proteins. Each CRISPR RNA (crRNA) produced from CRISPR transcripts is complementary to a target sequence and thus guides the Cas proteins to recognize and cleave the target in a sequence-specific manner [24]. Currently, CRISPR-Cas systems have been grouped into seven main types (types I-VI plus type U) based on the system complexity and signature proteins, that are classified into two classes (class 1 and class 2) [24-26]. Class 1 systems (containing types I, III, and IV) typically form multi-subunit protein-crRNA complex, whereas class 2 systems (containing types II, V, and VI) use a single crRNA-guided protein for target interference $[18,26]$. Functional CRISPR-Cas enzymes will introduce DNA injuries, e.g. double-stranded DNA breaks (DSB), within target sequences defined by guide RNAs (gRNA) [21, 23]. The resulting injuries will activate endogenous DNA repair mechanisms, such as nonhomologous end-joining (NHEJ) and homologous recombination (HR) [27, 28]. As most bacteria including $Z$. mobilis lack the efficient but error-prone NHEJ pathway, they repair DNA injuries rely largely on the HR in combination with donor templates. DNA repair through HR would give precise edits.

The type II CRISPR-Cas9 system from Streptococcus pyogenes has been exploited as a highly efficient genetic tool in various organisms for gene editing and regulation due to its simplicity and versatility $[20,29,30]$. However, the potential toxicity of Cas9 nuclease limited its broad application in certain prokaryotic hosts such as Corynebacterium glutamicum and Cyanobacteria sp. $[31,32]$. This would explain, at least partly, the fact that although this system has been employed for the native plasmid curing in Z. mobilis [33], no follow-up report on its application for genome engineering is accumulated in the literature.

Recently, Cas12a (Cpf1), a Type V RNA-programmable endonuclease, has also been characterized and engineered for genome editing [30,34,35], which was reported to have less toxicity to the prokaryotic cells compared to Cas9 $[24,31,32]$. Cas12a guided by a mature crRNA recognizes a protospacer flanked by a T-rich PAM (protospacer-adjacent motif) and creates staggered ends while Cas9 typically uses a G-rich PAM sequence for target discrimination and generates blunt ends [34, 36-40]. Collectively, Cas12a would be taken as an alternative or complement to Cas9 for prokaryotic engineering. Zetsche et al., applied in vitro PAM identification assay and confirmed the PAM for Cas12a as $5^{\prime}-\mathrm{TTN}$ [34]. Leenay et al. performed a comprehensive screening of PAM with the catalytically dead Cas12a indicated that PAM sequences of GTTC and TTTN yielded stronger repression in comparison to a non-targeting RNA control [37].

In this study, we aim to explore the possibility to develop a highly efficient, convenient genetic engineering 
tool for Z. mobilis using the CRISPR-Cas12a system to facilitate our understanding of this ethanologic bacterium and to expedite the practices on metabolic engineering and genome engineering.

\section{Results and discussions}

Construction and functionality of genomic integrated

\section{Cas12a in Z. mobilis ZM4}

We initially attempted to establish the CRISPR-Cas12abased toolkit by expressing both the Cas12a effector nuclease and the crRNA via a single plasmid. However, transformation with this plasmid could only generate few transformants with a very poor transformation efficiency (Additional file 1: Fig. S1), possibly due to its relatively large size and plasmid copy number effect, hence leading to the failure in genome editing. To address this issue, a recombinant strain (ZM4-Cas12a) was constructed by integrating a cas $12 a$-expressing cassette driven by the tetracycline-inducible promoter Ptet, together with a spectinomycin antibiotic marker for selection, into the ZMO0038 locus through homologous recombination (Fig. 1a).

In order to assist CRISPR-Cas12a-based genome editing in $Z$. mobilis, self-targeting plasmids were constructed to individually express crRNAs consisting of a 19-nt direct repeat (DR) and a 23-nt guide sequence. It was reported that this crRNA species exhibited optimal performance for genome editing [34]. Following this principle, two plasmids, pEZ15a-sgr-PS-Ldh and pEZ15a-sgr-MS-Ldh, were designed to respectively target a sequence on the coding or noncoding strand of a lactate dehydrogenase encoding gene ZMO1237 (ldh).

We next assayed the DNA cleavage activity of the exogenously introduced CRISPR-Cas12a system. To this end, the self-targeting plasmids were individually introduced into either ZM4 or ZM4-Cas12a cells. Notably, whereas transformation of ZM4 cells yielded a very high transformation efficiency reaching $10^{5}$ c.f.u/ $\mu$ g DNA, transformation of ZM4-Cas12a cells got a thousand folds lowered transformation efficiency (Fig. 1b). By contrast, transformation of the crRNA-expressing plasmid into ZM4 cells or the plasmid vector (pEZ-sgr) into ZM4-Cas12a obtained high efficiency. Interestingly, even in the absence of tetracycline, expression of crRNA targeting the $l d h$ gene in Z. mobilis still conferred a nearly $100 \%$ killing frequency, indicating a basal expression of Cas12a driven by Ptet at the condition without tetracycline induction as reported before [41]. Collectively, these results suggested that the CRISPR-Cas12a system can efficiently

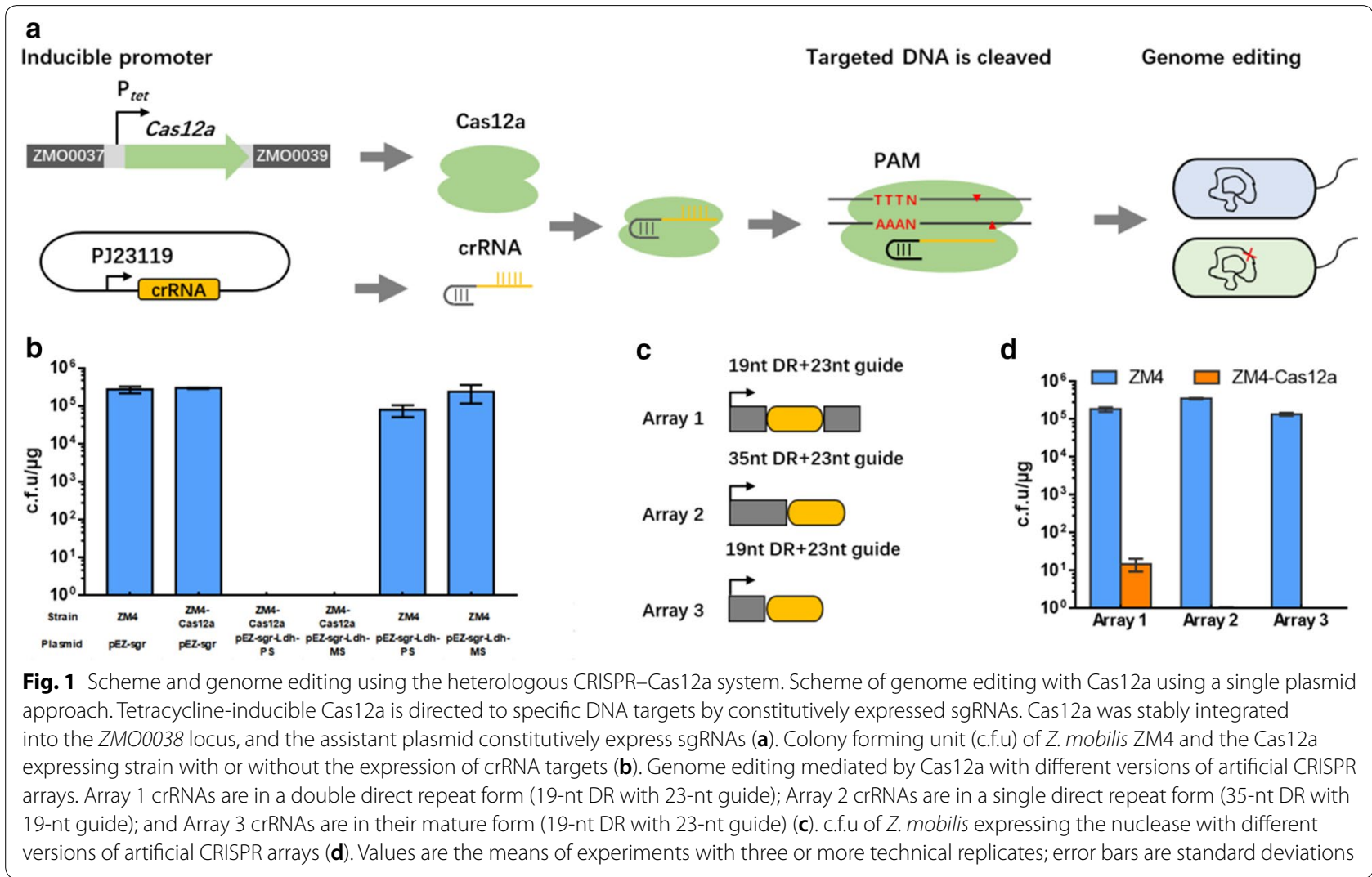


mediate targeted DNA cleavage, and the Cas12a expression alone in $Z$. mobilis is not toxic.

To further develop a compatible multiplex gene-editing strategy in Z. mobilis, we evaluated effects of crRNA variants on directing self-killing. The crRNAs variants all contain a 23-nt guide sequence targeting the $l d h$ locus of $Z$. mobilis genome but differed in DRs: in Array 1, the guide is sandwiched by two 19-nt DRs while in Array 2 and 3 only a downstream 35-nt or 19-nt repeat, respectively, is present (Fig. 1c). All the crRNAs efficiently directed the nuclease activity of the chromosomally integrated Cas12a to the target sequence for destruction, as almost no transformant could be obtained when the constructs expressing the crRNA variants were introduced into ZM4-Cas12a cells via electroporation (Fig. 1d). Few transformants were obtained from transformation with the Array 1-containing plasmid, which were subsequently identified to be escapers carrying a mutated Array 1 . Sanger sequencing results revealed that recombination occurred between the two 19-nt repeats, leading to the loss of the guide sequence.

\section{Application of CRISPR-Cas12a system for native plasmid curing in Z. mobilis}

Recently, a revised $Z$. mobilis ZM4 genome sequence $(\sim 2.06 \mathrm{Mb})$ was released, which includes four native plasmids named pZM32 (32,791 bp), pZM33 (33,006 bp), pZM36 (36,494 bp), and pZM39 (39,266 bp) according to their sequence sizes [9]. Sequencing analysis revealed that all four plasmids encode replicases that are required for their replication. Thus, if these replicase genes were inactivated using the CRISPR-Cas12a-based toolkit, the plasmid would lose the ability for replication and will then be consequently cured $[9,33]$.

Genes encode replicases for the native plasmids of pZM33, pZM36, and pZM39 are pZM33_028 (NZ CP023679), pZM36_036 (CP023680), and pZM39_032 (CP023681) respectively. gRNAs being designed to specifically target sequences within the replicase-encoding genes were expressed from the plasmids pEZ15a-sgr$\Delta$ pZM32, pEZ15a-sgr- $\Delta$ pZM33, pEZ15a-sgr- $\Delta$ pZM36 and pEZ15a-sgr- $\triangle \mathrm{pZM} 39$, respectively. $\mathrm{A}$ total of 200 300 ng of each plasmid DNA was transformed into ZM4-Cas12a. Primers specifically against each native plasmid were designed to further verify plasmid curing. Our results indicated that three native plasmids, pZM33, pZM36 and pZM39, were cured independently in one step with $100 \%$ efficiency (Fig. 2a), and the removal of these three native plasmids individually had no or negligible influence on cell growth (Fig. 2b).

Plasmids play pivotal role in the advancement of molecular biology, and various plasmid vectors for genetic and metabolic engineering purposes have been developed using an origin of replication region from native plasmids of $Z$. mobilis [42]. Eight $Z$. mobilis strains have been completely sequenced and contain 2-8 native plasmids with different sizes $[9,33]$. Plasmid stability and compatibility issues will occur when an engineered plasmid with the same origin of replication is introduced into the host strain, which will limit the application of this powerful tool for strain improvement [43]. The CRISPRCas12a-based toolkit established here for $Z$. mobilis was applied for native plasmid curing successfully, which will facilitate the investigation of plasmid gene function and future genome reduction of $Z$. mobilis.

\section{Application of CRISPR-Cas12a assisted ssDNA} recombineering for nucleotide substitutions in Z. mobilis

To introduce specific genomic changes, single strand oligonucleotide (ssDNA) with homology to the sequence flanking the DSB was used as templates to facilitate the repair of the damaged DNA. We tested this system by attempting to introduce point mutations into the $l d h$ gene using ssDNA oligonucleotide recombination. Mutation of targeting genome was designed to alter its seed sequence with two mismatching nucleotides, which will also generate a PstI restriction site. The recombinants with the engineered sequence can be checked by colony PCR and further verified by PstI digestion, such that the recombination efficiency can be evaluated.

Targeting plasmid pEZ-sgr-Ldh-PS ( $200 \mathrm{ng}$ ) was cotransformed with $1 \mu \mathrm{g}$ ssDNA editing template. There was an apparent strand bias for the editing efficiency. Targeting the lagging strand showed almost $100 \%$ editing efficiency, which was four folds higher than that of targeting the other (Fig. 3a). This result is consistent with previous reports in Mycobacterium smegmatis and C. glutamicum $[18,31]$. The length of editing template also affected the transformation efficiency with reduced colony forming unit (c.f.u), whereas it had litter effect on editing efficiency (Fig. 3b). These data indicated that the CRISPR-Cas12a-assisted ssDNA recombineering system can be served as a robust and precise tool for introducing minor nucleotide substitutions in $Z$. mobilis.

\section{Application of CRISPR-Cas12a system for gene deletion and replacement}

Homologous recombination (HR) can be utilized for accurate sequence modifications such as nucleotide substitutions or gene insertion when a homologous DNA donor template is provided in conjunction with the generation of the DSB [19]. Genome editing can therefore be initiated by generating site-specific DSB in the genome. In order to utilize HR for gene editing, a DNA repair template containing the desired sequence must be delivered into cells of interest with the crRNA and Cas12a. 

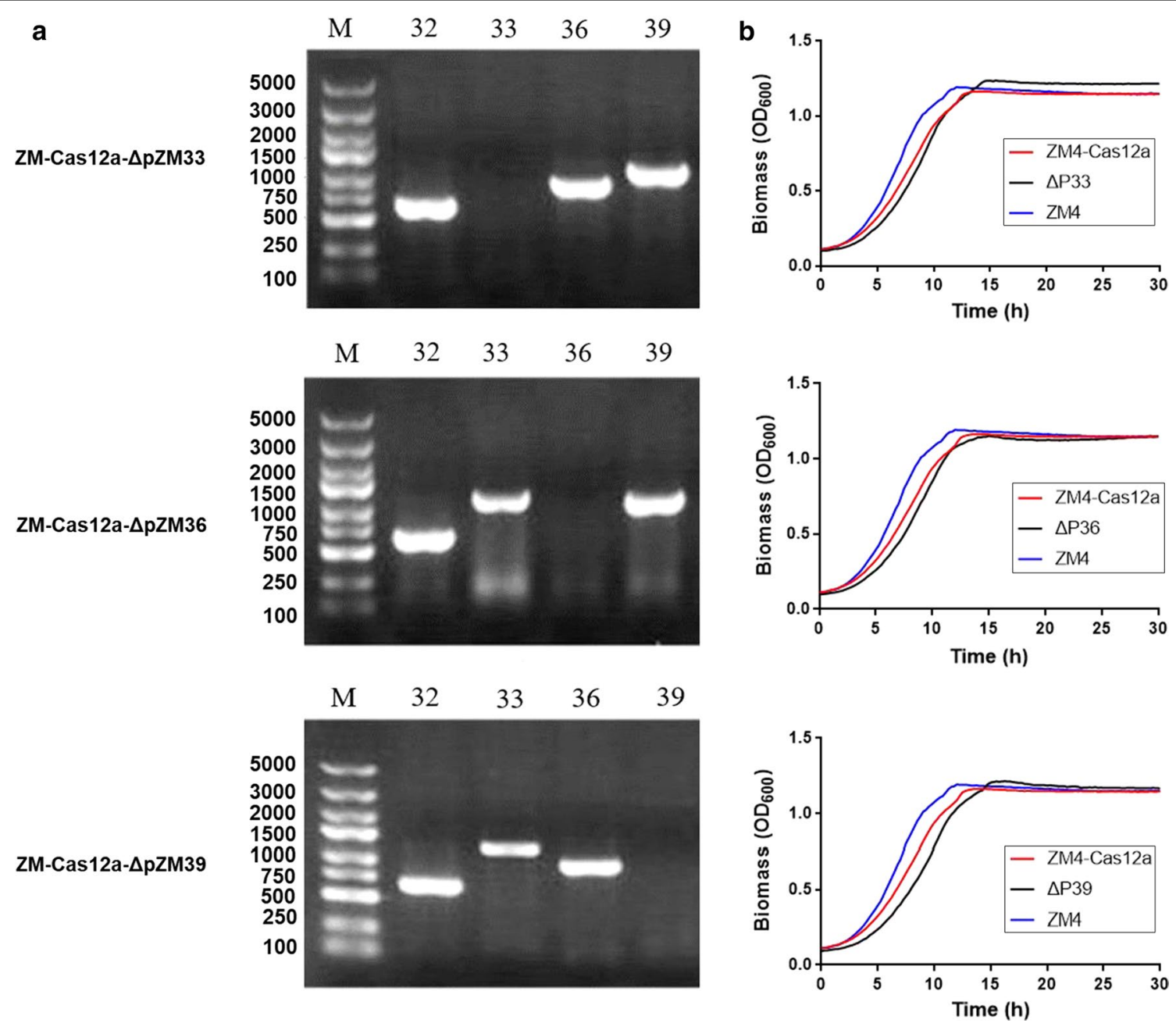

Fig. 2 Native plasmid curing in Z. mobilis by CRISPR-Cas12a system and the impact of plasmid curing on cellular growth. The native plasmid curing was identified by colony PCR. Primers specific for native plasmids of pZM33 (P33-Check-F/R, 1192 bp), pZM36 (P36-Check-F/R, 896 bp) and pZM39 (P39-Check-F/R, 1121 bp) were used to verify the existence of each plasmid, respectively (a). The growth curve of Z. mobilis and plasmid-deleted mutants using Bioscreen C (b)

The repair template contains the desired edit as well as additional homologous sequences flanking upstream and downstream of the target locus (Fig. 4a, b).

$Z M O 0028$ is a putative methylated adenine recognition and restriction gene belonging to the type IV R-M system, and the deficient of $Z M O 0028$ improved transformation efficiency of $Z$. mobilis [44]. ZMO0347 encodes the RNA chaperone $\mathrm{Hfq}$ involving in tolerance against multiple hydrolysate inhibitors such as acetate, vanillin, furfural, and HMF in Z. mobilis [45-47]. Since both genes are not essential for cell viability, gRNAs were designed to target the $Z M O 0028$ and the ZMO0347 loci for gene deletion using the CRISPR-Cas12a assisted genome-editing system developed in this work. A pair of homologous arms was selected from the flanking sequences of $Z M O 0028$ or
$Z M O 0347$ and cloned into the pEZ-sgr vector, generating the donor-carrying plasmid. The deletion efficiency of ZMO0028 or ZMO0347 reached $90-100 \%$ (Fig. 4e). The obtained recombinants were further identified by PCR (Fig. 4c), and the PCR product was confirmed by Sanger sequencing (Fig. 4d). Those results indicated that HR-induced precise mutagenesis is particularly useful for generating markrless deletion mutations.

In the case of gene replacement, gRNA was designed for generating DSB within the ZMO0028 locus. A reporter gene ( $m$ Cherry) driving by a constitutive promoter Pgap and a lactate dehydrogenase-encoding gene ldh from Bacillus coagulans ( $L d h B c$ ) under the control of the constitutive strong promoter $P a d h B$, sandwiched by two recombination arms respectively homologous to 

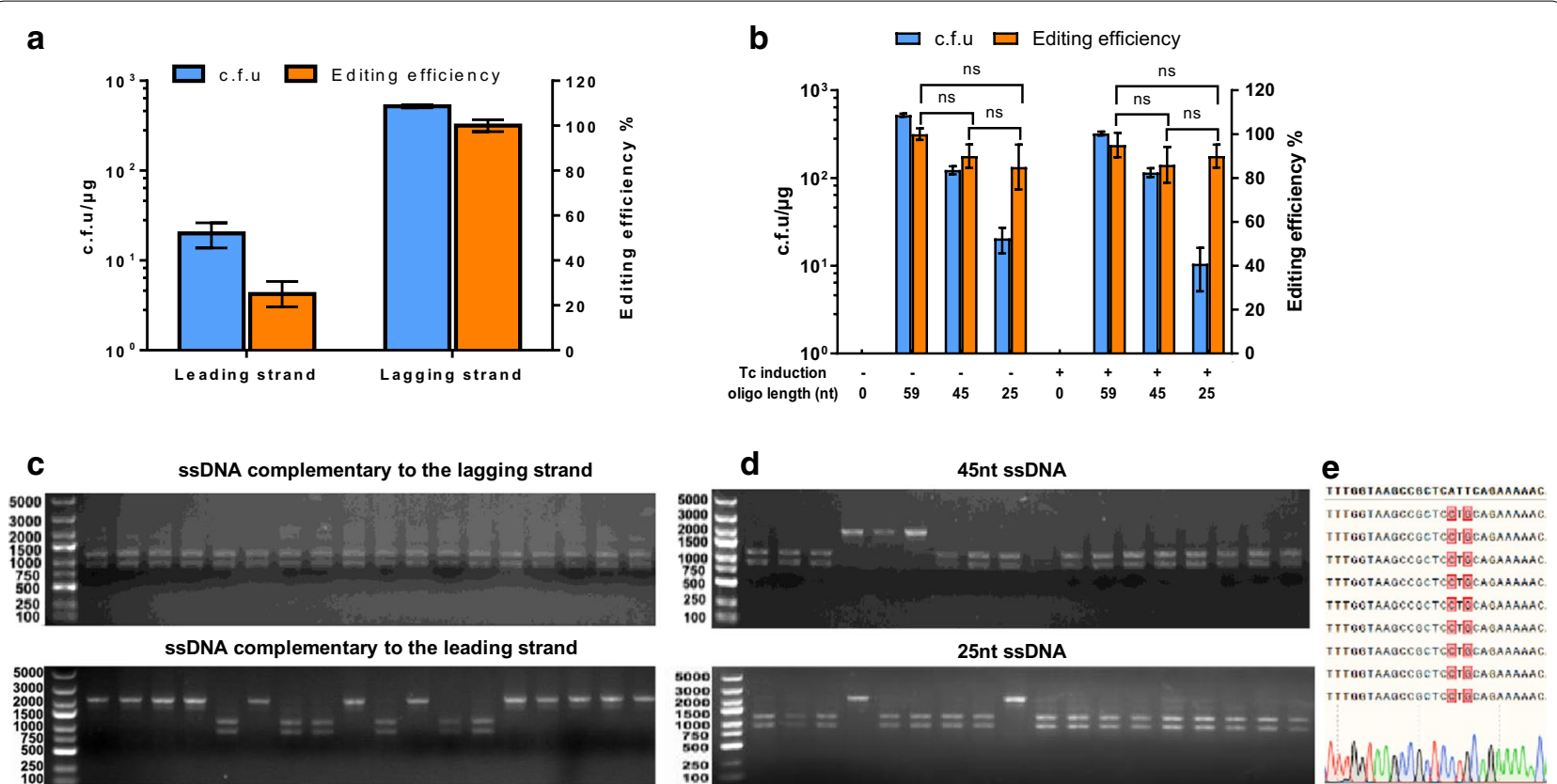

Fig. 3 The impact of crRNA on lagging and leading strand as well as the length of direct repeats in artificial CRISPR arrays on transformation and editing efficiencies of the CRISPR-Cas12a assisted ssDNA recombineering in Z. mobilis. c.f.u and editing efficiency of /dh after editing by $59 \mathrm{nt}$ template oligonucleotide targeting the lagging strand and leading strand, respectively (a), or by oligonucleotide template with different length targeting the lagging strand mediated by plasmid pEZ-sgr-ldh expressing /dh targeting crRNA (b). Transformants generated from CRISPR-Cas12a assisted ssDNA recombination targeting on lagging strand, leading strand (c), or oligonucleotide template with different length targeting the lagging strand (d). Transformants were screened by colony PCR with primer pair Ldh-check-F/R, and the PCR product was then digested with a Pstl to investigate the recombination efficiency. A $2.1 \mathrm{~kb}$ fragment indicates wild-type genotype, whereas the presence of 1.2 and $0.9 \mathrm{~kb}$ fragments indicate recombinant genotypes. The representative Idh recombinants were confirmed by Sanger sequencing (e). A multiple comparison between groups was performed for the editing efficiencies using different length of ssDNA through analysis of variance (ANOVA) followed by unpaired two-tailed student- $t$ test using GraphPad InStat software (GraphPad, Prism 8). P value $<0.05$ was considered to be statistically significant, and ns represents non-significant. Values are the means of experiment with three or more technical replicates, error bars are standard deviation

the up- or down-flanking sequences of the ZMO0028 locus, were cloned into the pEZ-sgr vector to generate the corresponding donor-carrying plasmids. Transformation with $1 \mu \mathrm{g}$ of $m$ Cherry crRNA specific plasmid into Z. mobilis competent cells produced more than 40 c.f.u., among which $95 \%$ (Fig. 4f) were correctly edited (Fig. 4c, d). The edited strain with mCherry gene driven by Pgap promoter inserted into ZMO0028 was then characterized by flow cytometry, and the fluorescence of recombinant strain showed a fluorescence shift compared with that of the wild-type strain (Fig. 4g).

\section{Characterization of a lactate-producing Z. mobilis strain constructed by the CRISPR-Cas 12a system}

Recombinant strain ZM-LdhBc harboring heterologous lactate gene developed in this study was then cultured in $100-\mathrm{mL}$ shake flasks with $80 \mathrm{~mL}$ RMG5 medium containing $50 \mathrm{~g} / \mathrm{L}$ glucose. The results exhibited that ZM-LdhBc had a significant improvement in lactate production with lactate titer reaching $2.12 \mathrm{~g} / \mathrm{L}$ and a correspondingly reduced ethanol production from 21.25 to $18.31 \mathrm{~g} / \mathrm{L}$ compared with the wild-type strain ZM4 (Fig. 5).

Those result thus demonstrated that the expression of a synthesized $L d h B c$ under the control of the constitutive strong promoter $P a d h B$ is crucial for lactate production. However, the final ethanol titer was similar between the wild type and mutant strain, which indicated that $L d h B c$ gene alone cannot effectively compete with ethanol production, and other strategy is needed to divert more carbon flux from ethanol production into lactate biosynthesis such as the knockout of pyruvate decarboxylase (PDC). However, PDC is essential for cell viability of the wild-type $Z$. mobilis and thus cannot be easily knocked out [48]. The high editing efficiency of CRISPR-Cas12a assisted ssDNA recombineering (ca. 100\%) discussed above indicated that site-directed mutagenesis of $p d C$ gene could be an alternative strategy to reduce the affinity of PDC to pyruvate and thus redirect the intermediate pyruvate for maximum lactate production. 


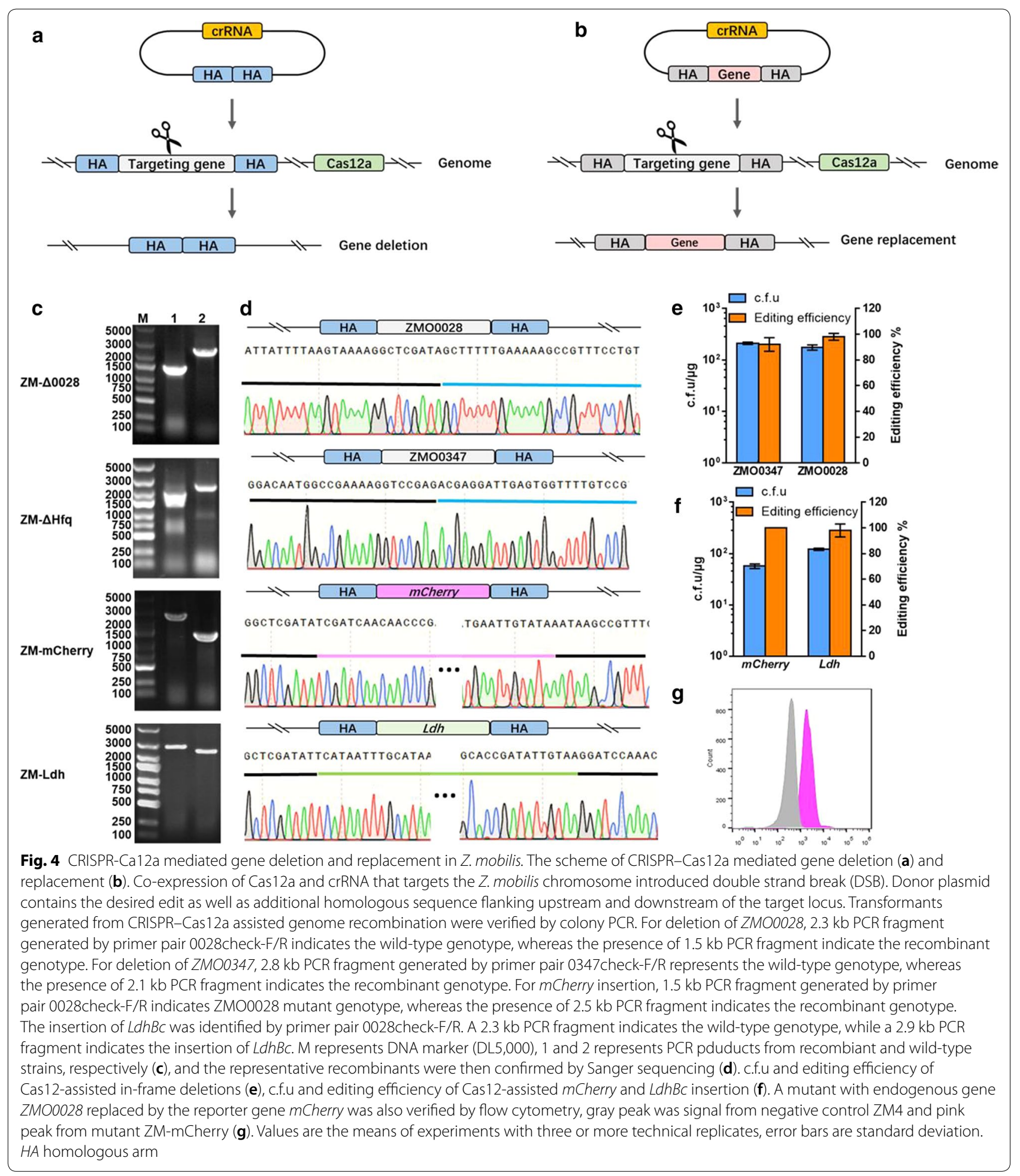

\section{Conclusion}

An efficient genome-editing tool based on the CRISPRCas12a was developed through the integration of Cas12a into the chromosome, and the impact of crRNA on lagging and leading strand as well as the length of direct repeats in artificial CRISPR arrays were further investigated to optimize the system, which was then successfully applied for plasmid curing, gene deletion 

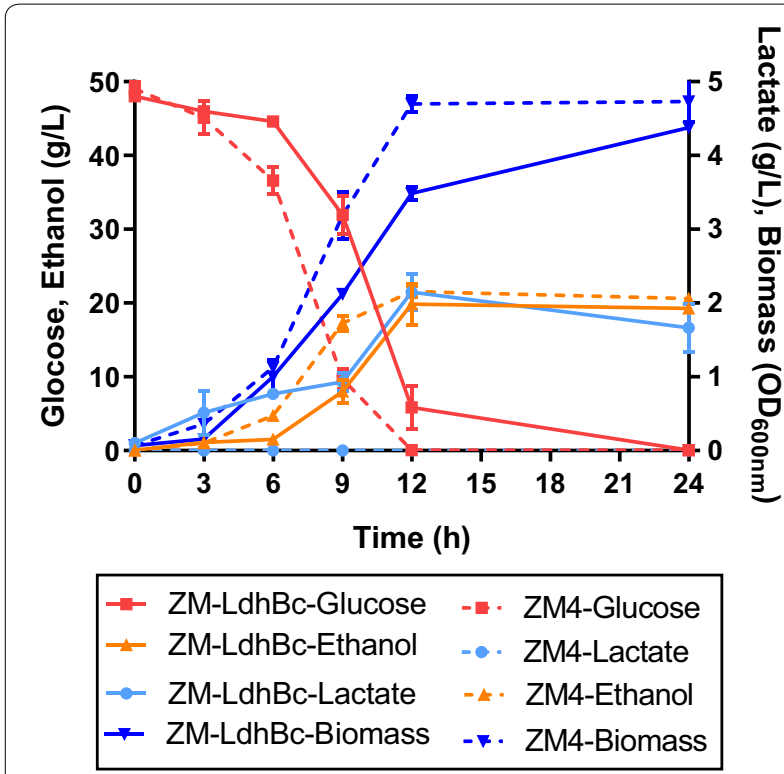

Fig. 5 Growth curve, glucose consumption as well as ethanol and lactate production in $\mathrm{ZM}-\mathrm{LdhBc}$. The titer $(\mathrm{g} / \mathrm{L}$ ) of major end products detected in the supernatant of wild-type ZM4 and lactate producing Z. mobilis strains. Biomass was evaluated by determination of optical density $\left(\mathrm{OD}_{600 \mathrm{~nm}}\right)$. Values are the means of experiment with three or more technical replicates, error bars are standard deviation

and insertion as well as nucleotide substitution. In addition, the CRISPR-Cas12a system applied in this study for Z. mobilis was also used for metabolic engineering practices with a lactate-producing recombinant strain developed. The successful demonstration of the application of CRISPR-Cas12a system in Z. mobilis will extend the existing genetic toolbox for metabolic engineering and genome engineering in $Z$. mobilis, and can also be served as an example of developing CRISPRCas12a genome-editing tools in other microorganisms.

\section{Materials and methods}

\section{Strains and culture conditions}

Escherichia coli DH5 $\alpha$ was used in this study for plasmid maintenance, which was cultured at $37^{\circ} \mathrm{C}$ in Luria-Bertani medium (LB, $10 \mathrm{~g} / \mathrm{L}$ tryptone, $5 \mathrm{~g} / \mathrm{L}$ yeast extract, $10 \mathrm{~g} / \mathrm{L} \mathrm{NaCl}$ ). Z. mobilis ZM4 was the parent strain for genetic modifications and was cultured at $30{ }^{\circ} \mathrm{C}$ in rich medium (RMG5: $10 \mathrm{~g} / \mathrm{L}$ yeast extract, $50 \mathrm{~g} / \mathrm{L}$ Glucose, $\left.2 \mathrm{~g} / \mathrm{L} \mathrm{KH}_{2} \mathrm{PO}_{4}\right)$. When required, antibiotics were added to the growth media at the following final concentrations: spectinomycin, $100 \mu \mathrm{g} / \mathrm{mL}$; chloramphenicol, $50 \mu \mathrm{g} / \mathrm{mL}$ for both E. coli and Z. mobilis. All Z. mobilis and derivative strains used in this study are listed in Table 1.

\section{DNA manipulation techniques}

All constructs used in the study are listed in Additional file 2: Table S1. Sequences of the primers, crRNAs, and oligonucleotides used in the study are listed in Additional file 3: Table S2. Plasmids and chromosomal DNA were extracted using AxyPrep kits (Corning, China). DNA polymerases used were PrimerSTAR (Takara, Japan) or Taq DNA polymerases (Tsingke, China). Restriction endonucleases and T4 DNA ligase were from Thermo Scientific (USA), and the isothermal assembly method was used in this work [35]. Gene deletions were confirmed by PCR and Sanger sequencing (Tsingke, China).

\section{Generation of Cas12a-Targeting gRNA constructs}

Plasmid pEZ-sgr was used as the source for crRNA guides in $Z$. mobilis. This plasmid is a derivative of pEZ15A that carries a minimal CRISPR array containing synthetic promoter PJ23119 and two BsaI restriction sites for easy cloning of new spacers. The synthetic promoter PJ23119 was used to transcribe target-specific sgRNA. The Cas12a-targeting gRNA sequence was annealed using two single-stranded oligonucleotides and ligated into $B s a I$-linearized pEZ-sgr.

Table 1 List of Z. mobilis strains used in this study

\begin{tabular}{|c|c|c|}
\hline Strains & Description & Note \\
\hline Z. mobilis ZM4 & Z. mobilis subsp. mobilis ZM4 (wild-type strain) & ATCC31821 \\
\hline ZM-Cas12a & $\begin{array}{l}\text { The Cas12a expressing cassette with spectinomycin resistance } \\
\text { integrated into ZMO0038 locus }\end{array}$ & This study \\
\hline ZM- $\triangle 0028$ & ZM-Cas12a with deletion of ZMO0028 & This study \\
\hline $\mathrm{ZM}-\triangle \mathrm{Hfq}$ & ZM-Cas12a with deletion of ZMO0347 & This study \\
\hline ZM-mCherry & ZMO0028 replaced by the reporter gene mCherry & This study \\
\hline ZM-LdhBc & ZMO0028 replaced by gene $L d h B C$ & This study \\
\hline ZM-Cas12a- $\triangle \mathrm{pZM} 33$ & ZM-Cas12a with native plasmid pZM33 cured & This study \\
\hline ZM-Cas12a- $\triangle \mathrm{pZM} 36$ & ZM-Cas12a with native plasmid pZM36 cured & This study \\
\hline ZM-Cas12a- $\Delta$ pZM39 & ZM-Cas12a with native plasmid pZM39 cured & This study \\
\hline
\end{tabular}


Since the PAM for Cas12a were reported to be $5^{\prime}$-TTN [34], and PAM GTTC and TTTN yielded stronger repression in comparison to a non-targeting RNA control [37]. Collectively, the PAM sequence $5^{\prime}$-TTTN$3^{\prime}$ was used in this study. Oligonucleotides for spacers were designed as follow: the coding region of the target genes were screened for the presence of a PAM of $5^{\prime}$-TTTN-3'. For every PAM found, 23-nt downstream sequence was selected as the potential target sequence, then oligonucleotides were designed as $5^{\prime}$-AGAT $+(\operatorname{tar}-$ get sequence)- $3^{\prime}$ and $5^{\prime}$-TGAC + (reverse complement of the target sequence) $-3^{\prime}$. A list of all spacers tested in this study is provided in Additional file 2: Table S2. In addition, the homologous regions flanking the editing sites were also included as a donor for homology dependent repair purposes. The customized specific plasmid was then transferred into Cas12a-expressing $Z$. mobilis strain ZM-Cas12a.

\section{Electroporation of Z. mobilis}

Electro-competent $Z$. mobilis was prepared as described before with slight modifications [48]. Briefly, a single colony was inoculated into 5-mL RMG5 media and grown without shaking at $30^{\circ} \mathrm{C}$ for $24 \mathrm{~h}$ as the seed culture. The seed culture was then transferred into the screw-cap bottle. Cell culture was placed on ice for $30 \mathrm{~min}$ and cells were collected by centrifuging when reached an $\mathrm{OD}_{600}$ value of 0.4-0.6. Cell pellets were washed once with ice-cold sterile water, re-centrifuged, and washed twice with pre-chilled sterilized $10 \%(\mathrm{v} / \mathrm{v})$ glycerol. These pellets were resuspended in $10 \%$ glycerol at a concentration approximately 1000 folds higher than the starting culture. Competent cells were stored at $-80^{\circ} \mathrm{C}$ as small aliquots.

Z. mobilis cells were transformed with plasmids by electroporation (Bio-Rad Gene Pulser, 0.1-cm gap cuvettes, $1.6 \mathrm{kV}, 200 \Omega, 25 \mu \mathrm{F})$. After electroporation, $1-\mathrm{mL}$ RM medium was added to the electroporation mixture and cells were recovered at $30{ }^{\circ} \mathrm{C}$ for $3-5 \mathrm{~h}$. The revived culture was plated on solid mating media containing appropriate antibiotics, and then incubated at $30{ }^{\circ} \mathrm{C}$ for $2-3$ days for transformation efficiency determination.

\section{Curing of targeting plasmids}

In order to cure the targeting plasmid, the transformant harboring the targeting plasmid after genome editing was inoculated into RMG5 medium without antibiotics selection pressure for 8 to $16 \mathrm{~h}$, which were then spread on RMG5 plates without antibiotic. Colonies were confirmed as targeting plasmid cured by determining their sensitivity to chloramphenicol.

\section{Identification of edited genes}

To identify positive clones with nucleotide substitutions of the ldh (ZMO1237) open reading frame, colony PCR was conducted using primers Ldh-check-F/R. PCR products were digested with PstI. To confirm the deletion (or the replacement) of native gene, colonies with $Z M O 0028$ deletion or replacement of either $L d h B c$ or $m$ Cherry were identified by colony PCR using primers 0028 check-F/R . Colonies with ZMO0347 ( $H f q)$ deletion were identified by PCR primers 0347 -check-F/R. Colonies with correct PCR product sizes were selected as candidates and confirmed by Sanger sequencing (Tsingke, China).

\section{Fluorescence measurements}

The protocol used for flow cytometry analysis of fluorescence was modified slightly from a previous study [41, 49]. Briefly, cells were washed with phosphate buffered saline (PBS) twice and then resuspended into PBS to a concentration of $10^{7}$ cells $/ \mathrm{mL}$. Cells were analyzed by flow cytometry using Beckman CytoFLEX FCM (Beckman Coulter, USA) with the PBS as the sheath fluid. The fluorescence of mCherry was excited with the $561 \mathrm{~nm}$ and detected with PC5.5 [50-52]. To avoid rare events which could affect the population distribution, at least 20,000 events of each sample were analyzed. Data were processed via FlowJo software (FlowJo, LLC, USA).

\section{Construction of a lactate producing strain, and the cultivation assay}

To construct a lactate producing strain, a heterologous lactate dehydrogenase from Bacillus coagulans $(L d h B c)$ was codon optimized and synthesized. The native promoter $P a d h B$ driving the alcohol dehydrogenase (ZMO1596) involved in ethanol production was used to stimulate the expression of $L d h B c$. The $L d h B c$ flanked $800 \mathrm{bp}$ upstream and downstream of ZMO0028 was cloned into the pEZ-sgr vector to generate the donor plasmid. A specific crRNA targeting ZMO0028 was designed and assembled to the donor plasmid. The resulted plasmid was induced to the ZM4-Cas12a through Cas12a-mediated genome editing. Recombinant strain was identified by PCR and confirmed by Sanger sequencing. The correct strain was named ZM-LdhBc.

The seed culture of ZM4 and lactate producing strain ZM-LdhBc were firstly revived from frozen glycerol stocks in $\mathrm{RM}$ at $30^{\circ} \mathrm{C}$ for $6 \sim 8 \mathrm{~h}$ without shaking. Then seed culture were transferred into $100-\mathrm{mL}$ shake flasks containing $80 \mathrm{~mL} \mathrm{RM}$ medium at an initial $\mathrm{OD}_{600} \approx 0.08$. Cultures were inoculated at $30{ }^{\circ} \mathrm{C}$ with a shaking speed of $100 \mathrm{rpm}$. Three replicates were used for each strain. Samples from the shake flasks were taken at various time points and the biomass was evaluated by determination 
of optical density $\left(\mathrm{OD}_{600 \mathrm{~nm}}\right)$ using spectrometer. Culture supernatants were collected and filtered through a $0.22 \mu \mathrm{m}$ filter for further analysis.

\section{High-pressure liquid chromatography (HPLC) analysis} High-pressure liquid chromatography (HPLC) was performed using a Shimadzu HPLC system (Japan) equipped with Aminex Resin-Based Columns (Bio-Rad) and refractive index detector (RID) to quantify glucose, ethanol, and lactate. The mobile phase is $5 \mathrm{mM} \mathrm{H}_{2} \mathrm{SO}_{4}$, and the flow rate was set as $0.5 \mathrm{~mL} / \mathrm{min}$ at $60^{\circ} \mathrm{C}$.

\section{Supplementary information}

Supplementary information accompanies this paper at https://doi. org/10.1186/s12934-019-1219-5.

Additional file 1: Figure S1. The transformation efficiency of Z. mobilis ZM4 and the Cas12a expressing strain with or without the expression of crRNA targets. ZM4 represented the wild-type strain Zymomonas mobilis ZM4, ZM4-Cas12a represented the recombinant strain with Cas12a integrated at the ZMO0038 locus of ZM4 genome. pEZ-sgr and its derivates containing crRNA scaffold, the pEZ-Cas12a expressing both the Cas12a effector nuclease and the crRNA via a single plasmid. Values are the means of experiments with three or more technical replicates; error bars are standard deviation.

Additional file 2: Table S1. List of plasmids used in this study.

Additional file 3: Table S2. Primers used in this study.

\section{Abbreviations}

Cas: CRISPR associated protein; CRISPR: Clustered Regularly Interspaced Short Palindromic Repeats; crRNA: CRISPR RNA; DR: direct repeat; DSB: doublestrand break; ED: Entner-Doudoroff pathway; FCM: flow cytometry; HPLC: high-pressure liquid chromatography; HR: homologous recombination; Ldh: lactate dehydrogenase; NHEJ: nonhomologous end-joining; PAM: protospacer adjacent motif; PDC: pyruvate decarboxylase; RID: Refractive index detector; sgRNA: single-strand guide RNA; ssDNA: single-stranded DNA.

\section{Acknowledgements}

We thank Yong Yang, and Yangyang Zhan from Hubei University for helpful discussion.

\section{Authors' contributions}

SY conceived and designed the experiments with inputs from all authors. WS constructed recombinant strain and plasmids, with help from JZ, MQ, BG, QY, WB, YX, YZ, and WP. WS and SY wrote the manuscript. All authors contributed to data analyses, read and revised. All authors read and approved the final manuscript.

\section{Funding}

This work was supported by the National Key Research and Development Program of China (2018YFA0900300), National Science Foundation of China (21978071 and U1932141), and the Technical Innovation Special Fund of Hubei Province (2019AHB055 and 2018ACA149). We also acknowledge the support from State Key Laboratory of Biocatalysis and Enzyme Engineering.

\section{Data availability}

The authors declare that all the data supporting the findings of this study are available within the paper and its Supplementary Information files or are available from the corresponding author on request.

\section{Ethics approval and consent to participate}

The authors declare that this study does not involve human subjects, human material and human data.
Consent for publication

All authors read and approved the final manuscript.

\section{Competing interests}

The authors declare that they have no competing interests.

Received: 1 July 2019 Accepted: 26 September 2019

Published online: 03 October 2019

\section{References}

1. Wang X, He Q, Yang Y, Wang J, Haning K, Hu Y, Wu B, He M, Zhang Y, Bao J, et al. Advances and prospects in metabolic engineering of Zymomonas mobilis. Metab Eng. 2018;50:57-73.

2. Yang S, Fei Q, Zhang Y, Contreras LM, Utturkar SM, Brown SD, Himmel ME, Zhang M. Zymomonas mobilis as a model system for production of biofuels and biochemicals. Microb Biotechnol. 2016;9(6):699-717.

3. Panesar PS, Marwaha SS, Kennedy JF. Zymomonas mobilis: an alternative ethanol producer. J Chem Technol Biot. 2006;81(4):623-35.

4. Wu B, Qin H, Yang Y, Duan G, Yang S, Xin F, Zhao C, Shao H, Wang Y, Zhu Q, et al. Engineered Zymomonas mobilis tolerant to acetic acid and low pH via multiplex atmospheric and room temperature plasma mutagenesis. Biotechnol Biofuels. 2019:12:10.

5. Zhang M, Eddy C, Deanda K, Finkelstein M, Picataggio S. Metabolic engineering of a pentose metabolism pathway in ethanologenic Zymomonas mobilis. Science. 1995;267(5195):240-3.

6. Xia J, Yang Y, Liu CG, Yang S, Bai FW. Engineering Zymomonas mobilis for robust cellulosic ethanol production. Trends Biotechnol. 2019:37(9):960-72.

7. Agrawal M, Wang Y, Chen RR. Engineering efficient xylose metabolism into an acetic acid-tolerant Zymomonas mobilis strain by introducing adaptation-induced mutations. Biotechnol Lett. 2012;34(10):1825-32.

8. Chacon-Vargas K, Chirino AA, Davis MM, Debler SA, Haimer WR, Wilbur JJ, Mo X, Worthing BW, Wainblat EG, Zhao S, et al. Genome sequence of Zymomonas mobilis subsp. mobilis NRRL B-1960. Genome Announc. 2017:5(30):e00562.

9. Yang S, Vera JM, Grass J, Savvakis G, Moskvin OV, Yang Y, Mcllwain SJ, Lyu Y, Zinonos I, Hebert AS, et al. Complete genome sequence and the expression pattern of plasmids of the model ethanologen Zymomonas mobilis ZM4 and its xylose-utilizing derivatives 8b and 2032. Biotechnol Biofuels. 2018;11:125.

10. Chen C, Wu L, Cao Q, Shao H, Li X, Zhang Y, Wang H, Tan X. Genome comparison of different Zymomonas mobilis strains provides insights on conservation of the evolution. PLOS ONE. 2018;13(4):e0195994.

11. Zhao N, Pan Y, Liu H, Cheng Z. Draft Genome Sequence of Zymomonas mobilis ZM481 (ATCC 31823). Genome Announc. 2016:4(2):e00193.

12. Kouvelis VN, Teshima H, Bruce D, Detter C, Tapia R, Han C, Tampakopoulou VO, Goodwin L, Woyke T, Kyrpides NC, et al. Finished genome of Zymomonas mobilis subsp. mobilis strain CP4, an applied ethanol producer. Genome Announc. 2014;2(1):e00845.

13. Zhao N, Bai $Y$, Zhao XQ, Yang ZY, Bai FW. Draft genome sequence of the flocculating Zymomonas mobilis strain ZM401 (ATCC 31822). J Bacteriol. 2012;194(24):7008-9.

14. Yang S, Pappas KM, Hauser LJ, Land ML, Chen GL, Hurst GB, Pan C, Kouvelis VN, Typas MA, Pelletier DA, et al. Improved genome annotation for Zymomonas mobilis. Nat Biotechnol. 2009;27(10):893-4.

15. Joung J, Konermann S, Gootenberg JS, Abudayyeh OO, Platt RJ, Brigham MD, Sanjana NE, Zhang F. Genome-scale CRISPR-Cas9 knockout and transcriptional activation screening. Nat Protoc. 2017;12(4):828-63.

16. Pappas KM, Galani I, Typas MA. Transposon mutagenesis and strain construction in Zymomonas mobilis. J Appl Microbiol. 1997;82(3):379-88.

17. Wu Y, Li T, Cao Q, Li X, Zhang Y, Tan X. RecET recombination system driving chromosomal target gene replacement in Zymomonas mobilis. Electron J Biotechnol. 2017;30:118-24.

18. Yan MY, Yan HQ, Ren GX, Zhao JP, Guo XP, Sun YC. CRISPR-Cas12a-Assisted recombineering in bacteria. Appl Environ Microbiol. 2017;83(17):e00947.

19. Zhang J, Hong W, Zong W, Wang P, Wang Y. Markerless genome editing in Clostridium beijerinckii using the CRISPR-Cpf1 system. J Biotechnol. 2018;284:27-30. 
20. Barrangou R, Doudna JA. Applications of CRISPR technologies in research and beyond. Nat Biotechnol. 2016;34(9):933-41.

21. Koonin EV, Makarova KS. CRISPR-Cas: an adaptive immunity system in prokaryotes. F1000 Biol Rep. 2009;1:95.

22. Marraffini LA. CRISPR-Cas immunity in prokaryotes. Nature. 2015;526(7571):55-61.

23. Sorek R, Lawrence CM, Wiedenheft B. CRISPR-mediated adaptive immune systems in bacteria and archaea. Annu Rev Biochem. 2013;82:237-66.

24. Yao R, Liu D, Jia X, Zheng Y, Liu W, Xiao Y. CRISPR-Cas9/Cas12a biotechnology and application in bacteria. Synth Syst Biotechnol. 2018;3(3):135-49.

25. Bayat H, Modarressi MH, Rahimpour A. The conspicuity of CRISPR-Cpf1 system as a significant breakthrough in genome editing. Curr Microbiol. 2018;75(1):107-15.

26. Mohanraju P, Makarova KS, Zetsche B, Zhang F, Koonin EV, van der Oost J. Diverse evolutionary roots and mechanistic variations of the CRISPR-Cas systems. Science. 2016;353(6299):5147.

27. Marchisio MA, Huang Z. CRISPR-Cas type II-based synthetic biology applications in eukaryotic cells. RNA Biol. 2017;14(10):1286-93.

28. Su T, Liu F, Gu P, Jin H, Chang Y, Wang Q, Liang Q, Qi Q. A CRISPR-Cas9 assisted non-homologous end-joining strategy for one-step engineering of bacterial genome. Sci Rep. 2016;6:37895.

29. Shmakov S, Smargon A, Scott D, Cox D, Pyzocha N, Yan W, Abudayyeh OO, Gootenberg JS, Makarova KS, WolfYl, et al. Diversity and evolution of class 2 CRISPR-Cas systems. Nat Rev Microbiol. 2017;15(3):169-82.

30. Peters Jason M, Colavin A, Shi H, Czarny Tomasz L, Larson Matthew H, Wong S, Hawkins John S, Lu Candy HS, Koo B-M, Marta E, et al. A comprehensive, CRISPR-based functional analysis of essential genes in bacteria. Cell. 2016;165(6):1493-506.

31. Jiang Y, Qian F, Yang J, Liu Y, Dong F, Xu C, Sun B, Chen B, Xu X, Li Y, et al. CRISPR-Cpf1 assisted genome editing of Corynebacterium glutamicum. Nat Commun. 2017:8:15179.

32. Ungerer J, Pakrasi HB. Cpf1 is a versatile tool for CRISPR genome editing across diverse species of Cyanobacteria. Sci Rep. 2016;6:39681.

33. Cao QH, Shao HH, Qiu H, Li T, Zhang YZ, Tan XM. Using the CRISPR/Cas9 system to eliminate native plasmids of Zymomonas mobilis ZM4. Biosci Biotechnol Biochem. 2017;81(3):453-9.

34. Zetsche B, Gootenberg JS, Abudayyeh $\mathrm{OO}$, Slaymaker IM, Makarova KS, Essletzbichler P, Volz SE, Joung J, van der Oost J, Regev A, et al. Cpf1 is a single RNA-guided endonuclease of a class 2 CRISPR-Cas system. Cell. 2015;163(3):759-71.

35. Yamano T, Nishimasu H, Zetsche B, Hirano H, Slaymaker IM, Li Y, Fedorova I, Nakane T, Makarova KS, Koonin EV, et al. Crystal structure of Cpf1 in complex with guide RNA and target DNA. Cell. 2016;165(4):949-62.

36. Gao P, Yang H, Rajashankar KR, Huang Z, Patel DJ. Type V CRISPR-Cas Cpf1 endonuclease employs a unique mechanism for crRNA-mediated target DNA recognition. Cell Res. 2016;26(8):901-13.

37. Leenay RT, Maksimchuk KR, Slotkowski RA, Agrawal RN, Gomaa AA, Briner $A E$, Barrangou R, Beisel $C L$. Identifying and visualizing functional PAM diversity across CRISPR-Cas systems. Mol Cell. 2016;62(1):137-47.

38. Swarts DC, van der Oost J, Jinek M. Structural basis for guide RNA processing and seed-dependent DNA targeting by CRISPR-Cas12a. Mol Cell. 2017;66(2):221-33.
39. Woolston BM, Emerson DF, Currie DH, Stephanopoulos G. Rediverting carbon flux in Clostridium lijungdahlii using CRISPR interference (CRISPRi). Metab Eng. 2018:48:243-53.

40. Zhang X, Wang J, Cheng Q, Zheng X, Zhao G, Wang J. Multiplex gene regulation by CRISPR-ddCpf1. Cell Discov. 2017;3:17018.

41. Yang Y, Shen W, Huang J, Li R, Xiao Y, Wei H, Chou YC, Zhang M, Himmel $\mathrm{ME}$, Chen $\mathrm{S}$, et al. Prediction and characterization of promoters and ribosomal binding sites of Zymomonas mobilis in system biology era. Biotechnol Biofuels. 2019;12:52.

42. Browne GM, Skotnicki ML, Goodman AE, Rogers PL. Transformation of Zymomonas mobilis by a hybrid plasmid. Plasmid. 1984;12(3):211-4.

43. Talas A, Kulcsar PI, Weinhardt N, Borsy A, Toth E, Szebenyi K, Krausz SL, Huszar K, Vida I, Sturm A, et al. A convenient method to pre-screen candidate guide RNAs for CRISPR/Cas9 gene editing by NHEJ-mediated integration of a'self-cleaving' GFP-expression plasmid. DNA Res. 2017;24(6):609-21.

44. Kerr AL, Jeon YJ, Svenson CJ, Rogers PL, Neilan BA. DNA restrictionmodification systems in the ethanologen, Zymomonas mobilis ZM4. Appl Microbiol Biotechnol. 2011;89(3):761-9.

45. Wang $X$, He Q, Yang Y, Wang J, Haning K, Hu Y, Wu B, He M, Zhang Y, Bao J, et al. Advances and prospects in metabolic engineering of Zymomonas mobilis. Metab Eng. 2018;50:57-73.

46. Yang S, Pelletier DA, Lu TY, Brown SD. The Zymomonas mobilis regulator hfa contributes to tolerance against multiple lignocellulosic pretreatment inhibitors. BMC Microbiol. 2010;10(1):1-11.

47. Yang S, Tschaplinski TJ, Engle NL, Carroll SL, Martin SL, Davison BH, Palumbo AV, Rodriguez M Jr, Brown SD. Transcriptomic and metabolomic profiling of Zymomonas mobilis during aerobic and anaerobic fermentations. BMC Genomics. 2009;10:34.

48. Yang S, Mohagheghi A, Franden MA, Chou YC, Chen X, Dowe N, Himmel ME, Zhang M. Metabolic engineering of Zymomonas mobilis for 2,3-butanediol production from lignocellulosic biomass sugars. Biotechnol Biofuels. 2016;9(1):189.

49. Hakkila K, Maksimow M, Rosengren A, Karp M, Virta M. Monitoring promoter activity in a single bacterial cell by using green and red fluorescent proteins. J Microbiol Methods. 2003;54(1):75-9.

50. Chudakov DM, Matz MV, Lukyanov S, Lukyanov KA. Fluorescent proteins and their applications in imaging living cells and tissues. Physiol Rev. 2010;90(3):1103-63.

51. Shaner NC, Campbell RE, Steinbach PA, Giepmans BN, Palmer AE, Tsien RY. Improved monomeric red, orange and yellow fluorescent proteins derived from Discosoma sp. red fluorescent protein. Nat Biotechnol. 2004;22(12):1567-72.

52. Telford WG, Hawley T, Subach F, Verkhusha V, Hawley RG. Flow cytometry of fluorescent proteins. Methods. 2012;57(3):318-30.

\section{Publisher's Note}

Springer Nature remains neutral with regard to jurisdictional claims in published maps and institutional affiliations.

Ready to submit your research? Choose BMC and benefit from

- fast, convenient online submission

- thorough peer review by experienced researchers in your field

- rapid publication on acceptance

- support for research data, including large and complex data types

- gold Open Access which fosters wider collaboration and increased citations

- maximum visibility for your research: over $100 \mathrm{M}$ website views per year

At BMC, research is always in progress.

Learn more biomedcentral.com/submissions 